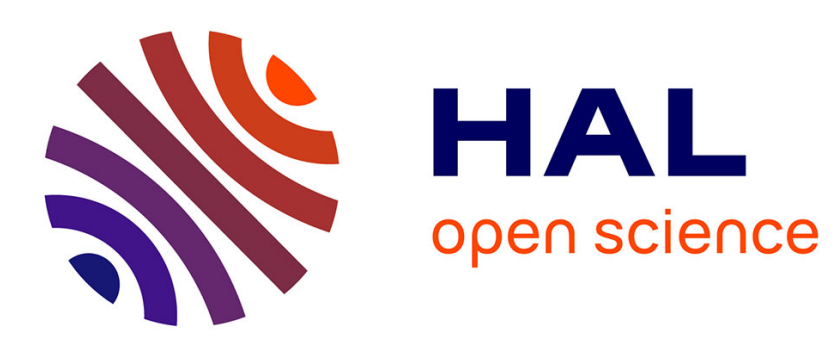

\title{
Energy harvesting in aeronautics for battery-free wireless sensor networks
}

Jean-Marie Dilhac, Marise Bafleur

\section{To cite this version:}

Jean-Marie Dilhac, Marise Bafleur. Energy harvesting in aeronautics for battery-free wireless sensor networks. IEEE Aerospace and Electronic Systems Magazine, 2014, 29 (8), pp.18-22. 10.1109/MAES.2014.130002 . hal-01064311

\section{HAL Id: hal-01064311 \\ https://hal.science/hal-01064311}

Submitted on 16 Sep 2014

HAL is a multi-disciplinary open access archive for the deposit and dissemination of scientific research documents, whether they are published or not. The documents may come from teaching and research institutions in France or abroad, or from public or private research centers.
L'archive ouverte pluridisciplinaire HAL, est destinée au dépôt et à la diffusion de documents scientifiques de niveau recherche, publiés ou non, émanant des établissements d'enseignement et de recherche français ou étrangers, des laboratoires publics ou privés. 


\title{
Energy Harvestings in Aeronautics for Battery-free Wireless Sensor Networks
}

\author{
Jean-Marie Dilhac $^{1,2}$, Marise Bafleur ${ }^{1}$ \\ ${ }^{1}$ Université de Toulouse, LAAS-CNRS \\ ${ }^{2}$ Université de Toulouse, INSA \\ Toulouse, France \\ dilhac@laas.fr,marise@laas.fr
}

\begin{abstract}
In this paper we present hands-on experience related to on-going - or prospective - implementations in aircrafts of systems for capturing, managing and storing primary energy from the environment. Primary energies such as thermal gradients and solar light will be illustrated through various scenarios: health monitoring of Aft Pylon Fairing, aerodynamic flight tests, or deployment of accelerometers.
\end{abstract}

Energy harvesting; thermo generation; photovoltaic; structural health monitoring; flight test

\section{INTRODUCTION}

In recent years, Wireless Sensor Networks (WSN) have been considered for various aeronautical applications, among others Structural Health Monitoring (SHM) and flight tests. Each network node would allow performing sensing, data processing and wireless transmission of information. Thus WSN permit (often new) functions without the need of adding extra wiring to an already large burden.

However, these nodes need to be self-powered, many of the advantages of wireless sensor networking being lost if external (i.e. wired) power sources were used. For this purpose, batteries offer a high energy density at low cost. However, there are critical drawbacks associated with the use of batteries: first environmental concerns and economical aspects associated with the replacement of primary batteries. Second, batteries may be prohibited since the wireless nodes are often implemented in locations without temperature regulation that could result both in a drastically reduced yield together with safety issues. Fortunately, batteries can be avoided through the use of environmental energy capture allowing a solution for long term, deploy and forget, WSN. However, environmental energy transducers, unlike batteries, are not energy reservoirs, and energy buffer storage is mandatory whatever the context.

In this paper we present hands-on experience related to ongoing - or prospective - implementations of energy harvesters in airliners.

In the first section, we will explain the reasons justifying the choice of ambient energy capture to power wireless sensor networks in an aircraft. Then we will briefly present the available sources of energy. In the last section, we will concentrate on examples of practical implementations.

\section{WHY ENERGY CAPTURE?}

Fig. 1 presents a decision tree summarizing the various issues to be raised when deploying a WSN on-board an aircraft.

The first step is to determine whether a Sensor Network is mandatory. It has to be kept in mind that most developments required for a given application, such as conceiving an energy management system, may be of little use for other applications, these customized developments being tightly bonded to the initial use. Consequently the decision of devising and deploying a WSN needs careful consideration.

In a second step, one has to determine whether the network needs to be wireless. In Fig. 2 the total electric cable lengths implemented in a car, a jet fighter and an airliner are compared. These lengths are also converted in kilometer per unit length of the car/aircraft as a measurement of complexity. From Fig. 2, it appears that fighter jets and airliners are similarly complex objects considering wiring, thus praising for wireless solutions when the deployment of new electronic sensors is to be considered. As a consequence of such a choice, measurement data need of course to be transmitted wirelessly; in addition the device has to be autonomous in energy. Specific components are therefore required to allow for this autonomy. Nevertheless, if stringent weight constraints (common in aeronautics) cannot afford these extra components, and if a power line runs close by, a connection to this line will be preferred.

Considering that a wireless implementation is chosen, the simplest way of gaining autonomy in energy is to use an energy reservoir such as a primary electrochemical battery. Such batteries are products of a mature industry: they offer a high energy density - and even a high power density when in parallel with an ultracapacitor - together with a low selfdischarge. Unfortunately these performances are strongly altered by the harsh environment (temperature mainly) encountered in most areas of an aircraft. Moreover, safety considerations, and the economic burden linked to the replacement of empty batteries, often preclude their large deployment.

The authors' work on energy harvesting in aircrafts has been partially supported by Fondation de Recherche pour l'Aéronautique et l'Espace, by Région Midi-Pyrénées, by Conseil pour la Recherche Aéronautique Civile, by Direction Générale de l'Armement and by Airbus. A preliminary version of this text was presented at More Electrical Aircraft Conference 2012. 


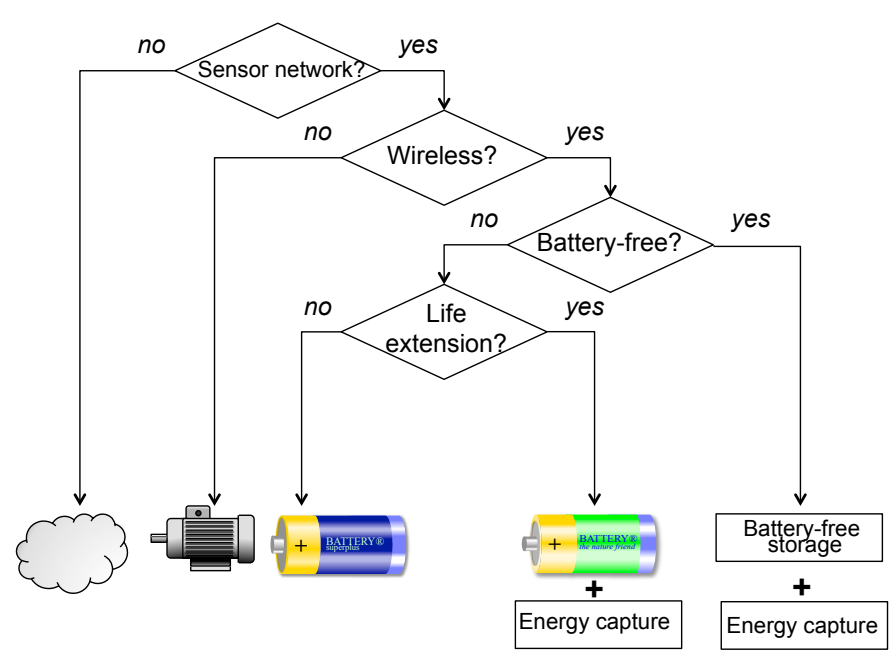

Figure 1. Energy decision tree.

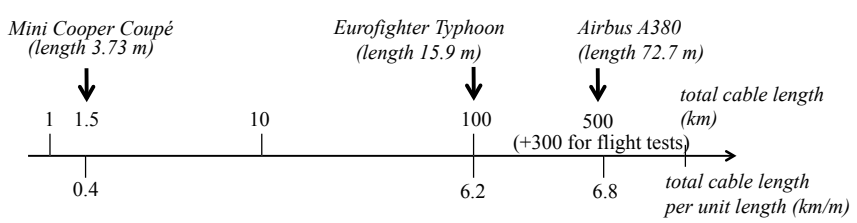

Figure 2. Wiring complexity for various means of transportation (estimations)

Energy capture from the environment is then mandatory, optionally coupled with the use of a secondary battery. Nevertheless, secondary batteries suffering from the same (or worse) drawbacks as primary ones, in the following, we will restrict ourselves to pure energy harvesting from the environment.

It is worth mentioning that energy storage is here still required for two reasons: first to accommodate for transient power surges from the load exceeding the mean electrical harvested power, and then to maintain the energetic autonomy in the case of an intermittent environmental source. Ultracapacitors may do the job; while being affected by extreme ambient temperatures similarly to batteries, they fortunately provide an infinite lifetime, they are safe and environment friendly.

\section{ENERGY HARVESTING}

Within an aircraft very different primary energy sources may be harvested, among others:

- Solar and indoor light.

- Transient and permanent thermal gradients.

- Mechanical movements, mechanical vibrations, acoustic noise.

\section{- Electromagnetic fields.}

Each category requires specific energy transducers. These transducers may directly benefit from long established R\&D and mature industry (photovoltaic); alternatively they may derive from different and older applications (thermoelectricity), there again benefiting from a strong industrial know-how.
Finally some very specific conversion principles are more recent (among others, piezoelectric micromechanical electrical systems using cantilevers, or devices capturing the energy of RF waves).

Generally speaking, such characteristics as the presence of moving parts, anisotropy, bandwidth, yield, and robustness to harsh environments are crucial parameters, when an alternative is possible.

In that context, thermoelectricity and photovoltaic are key technologies, and in the following we will restrict ourselves to these methods. However, we will not develop the theory or technology aspects, but concentrate on some practical considerations linked to the implementation of such systems.

\section{A. Thermo Generation and Aircrafts}

In an aircraft, thermal gradients - most of them being permanent during a flight - may originate from hot sources such as engines, auxiliary power unit (APU), bleed system, electrical or hydraulic actuators, electrical and electronics systems. They may also originate between thermally regulated and pressurized area (passenger cabin) and outside air.

To the knowledge of the authors, in most situations where thermo generation has been considered, the heat flux is first transferred into a Thermoelectric Generator (TEG) by solid conduction, the TEG being in contact with a hot or cold body, and then exits the TEG to cooler or hotter air by convection through a radiator. The TEG role is to convert a thermal flux into electrical energy; the conversion principle is based on the Seebeck effect and requires both cold and hot bodies. One may consider as an illustration a TEG affixed to the cold inner passenger cabin wall, and exchanging with the inside hotter cabin air (thermally regulated).

Conversely, outside pressurized zones, the heat flux may be reversed, the air being cooler than the aircraft frame. It is worth to mention that convection is there affected by two conflicting parameters: aircraft speed (possibly inducing an air flux fostering convection) and altitude (reducing atmospheric pressure and hence convection efficiency).

In the following of this paragraph, we will illustrate two schemes: exchanges first with outside air, and then with a hot source.

\section{1) Exchanging with outside air}

Outside pressurized volumes, transient thermal gradients take place when cruise level is changing, mainly during takeoff / climb, and descent / landing phases. They are due to the strong temperature dependence of air vs. altitude. They can be amplified by using Phase Change Materials (PCM) acting alternatively as the hot (climb) or cold (descent) body [1,2], transforming temporal temperature variations into (transient) spatial differences (Fig. 3.a). Unfortunately this gradient vanishes during cruise when all parts are more or less in thermal equilibrium, but they offer the possibility of transient thermal energy scavenging in areas where no hot source exists. In Fig. 3.b the thermal gradient obtained with such an arrangement is given for a short-haul flight. The PCM is here water whose freezing and liquefaction phases are clearly visible. It is worth mentioning that the use of a PCM, apart 
from increasing the thermal gradient by itself, consequently fosters TEG yield.

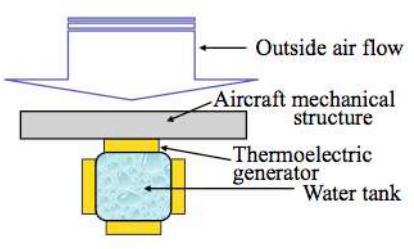

(a)

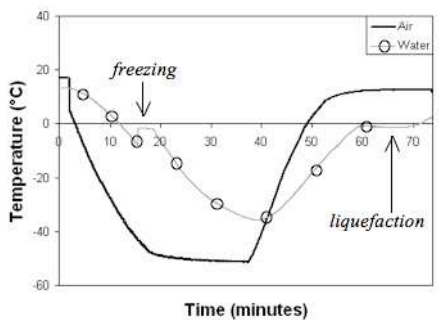

(b)
Figure 3. (a) schematic of TEG implementation (b) temperatures recorded during flight simulation in a climatic chamber. For $10 \mathrm{~g}$ of water, the harvested electrical energy was $34 \mathrm{~J}$ (from [1]).

The impact of using PCM to increase gradients is obvious when one considers the results of Figure 3.b where the gradient peak value exceeds $40^{\circ} \mathrm{C}$. The captured electrical energy is $34 \mathrm{~J}$ for a one-hour flight, translating into a mean harvested power of about $10 \mathrm{~mW}$. However, here the gradients are not permanent, and longer flights would give a lower power.

Permanent gradients are considered in the measures of Fig. 4. There, a TEG was affixed to the (cold) inner wall of an airliner hatch, and was exchanging with the inside nonpressurized air. In such a situation, TEGs could power 3D accelerometers to detect shocks or door position [3]. From figure 4 , it appears that the inside air was slightly hotter than outside atmosphere, however providing a relatively small gradient. This test was repeated on various aircraft doors and hatches, giving similar or unfortunately smaller gradients, except for passenger cabin doors where gradients were larger.

Comparing the above two sets of results, it appears that the use of PCM to increase gradients makes sense for short-haul flights, but is of little effect during cruise and consequently for long-haul flights. During cruise at constant altitude, unfortunately only modest gradients spontaneously develop when far from a hot source.

2) Exchanging with a permanent hot source: implementation in the aft pylon fairing

SHM of aircrafts consists in permanently monitoring key parameters to assess ageing effects. This is a major challenge with the objective to replace scheduled maintenance by predictive maintenance, therefore reducing costs. This may also reduce exploitation costs by reducing mechanical safety margins and consequently aircraft weight and fuel consumption [4]. SHM is a key player for airlines facing the "continual challenges of maximizing asset utilization at low operating cost and maximum revenue.’[5]
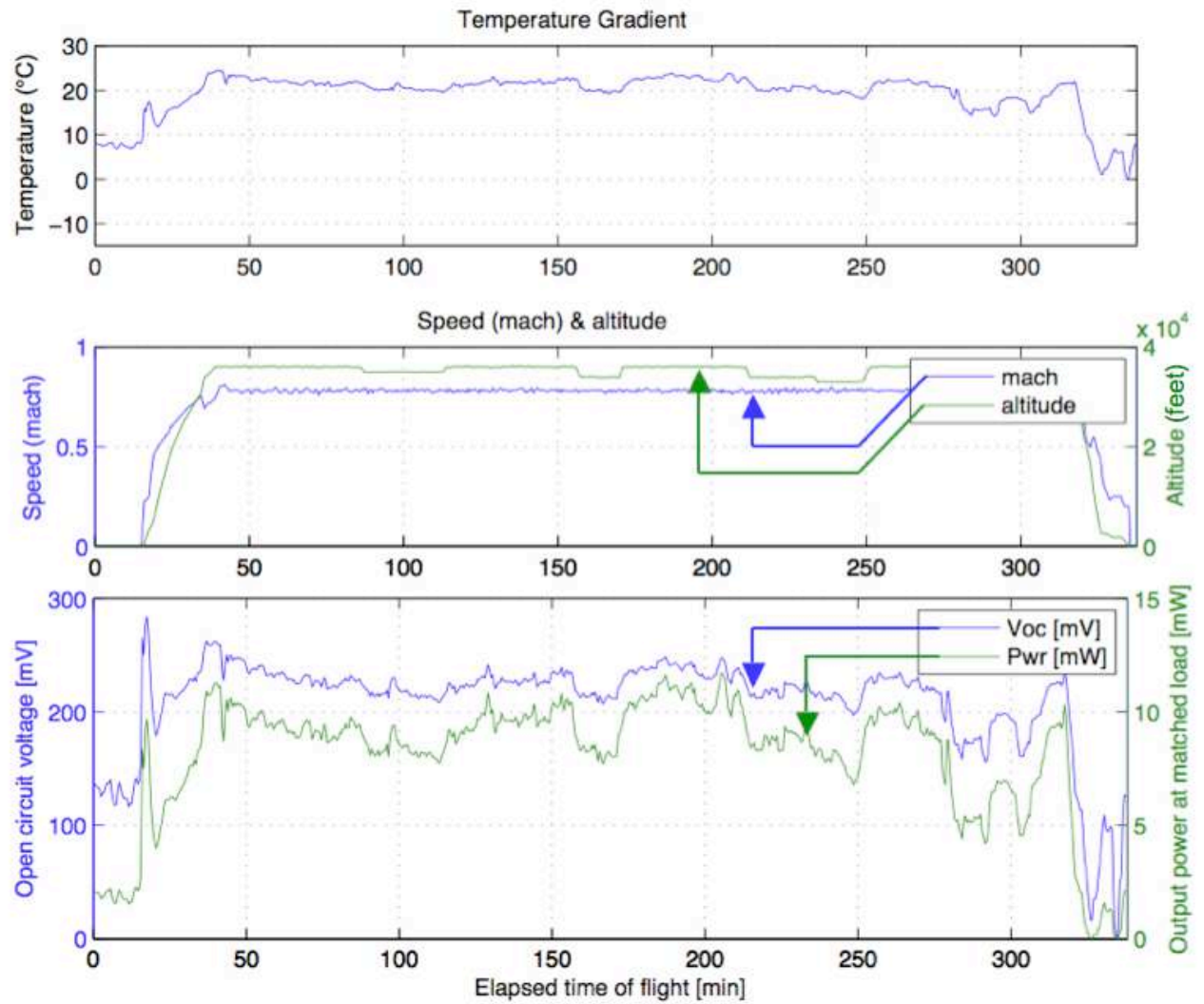

Figure 4. From top to bottom: experimental temperature gradient in the surrounding of an airliner hatch, flight level and speed vs. time. Electrical outputs (open circuit voltage $\mathrm{V}_{\mathrm{oc}}$, and output power at matched load $\mathrm{P}_{\mathrm{wr}}$ ) computed for a Micropelt TE-POWER PLUS TEG (from [3]). 
Commercial wired sensor networks are already being proposed for this purpose, at least for early detection of cracks within the metal structure of military aircrafts being operated for many more years than originally planned [6].

In that context, we have had the opportunity to work on the Aft Pylon Fairing (APF) of an airliner, for the implementation of a WSN for SHM. APF is an important device whose task is to reduce drag and to protect the pylon primary and secondary rear structures from extreme temperature (see Fig. 5). It is itself submitted to strong sonic load, high mechanical vibration levels and extreme temperatures (hundreds of ${ }^{\circ} \mathrm{C}$ for some areas). It is therefore a piece of choice for SHM.

Considering energy harvesting, the permanent thermal gradients developing during cruise between APF walls and inner air, look like an ideal source of energy. However, apart from the fact that the devices used for energy capture will have to qualify vs. very stringent aeronautical requirements, sonic load is here a very specific and robustness-demanding parameter. As a consequence, appropriate acoustic tests up to levels above $150 \mathrm{dBa}$, and a special certification process are mandatory in addition to classical certification.

However, above all, the final result of the holistic design of a comprehensive WSN node may also be quite surprising as it is illustrated in Fig. 6 where the main functions of a node are considered: sensing, energy harvesting and storage, energy management and signal processing.

On this figure, the parabolic curve represents the output power of a TEG vs. the temperature of the wall to which it is bonded. For the purpose of illustration, the inner air temperature is supposed to be $100^{\circ} \mathrm{C}$ and the targeted power output is supposed to be at least $17 \mathrm{~mW}$. Consequently the TEG must be affixed in areas where the wall temperature is at least $160^{\circ} \mathrm{C}$.

On the other hand, the sensor monitoring the parameter used for SHM answers to other requirements, such as a location near the mechanical part to be monitored. For obvious reasons, this location is likely to be close to the hottest zones of the APF, and for the purpose of demonstration this temperature will be there supposed to be above TEG maximum operating temperature ${ }^{1}$. Therefore, TEG and sensor cannot be affixed together and will need to be wired to each other.

Similar considerations for the electronics and energy storage devices lead to the conclusion that, even if the Sensor Network is macroscopically wireless, it is locally necessarily wired, as a result of the harsh environment.

This illustrates the unorthodox fact that for such harsh environments, the usual scheme in which all functions are integrated together (all-in-one topology) is no longer true and that a split topology is mandatory (see Fig. 7). One may even consider a single TEG implemented in an optimized location delivering power to close by devices.

Examining an assortment of TEGs from about twelve different manufacturers, the authors have noticed maximum operating temperatures ranging from 100 to $280^{\circ} \mathrm{C}$.

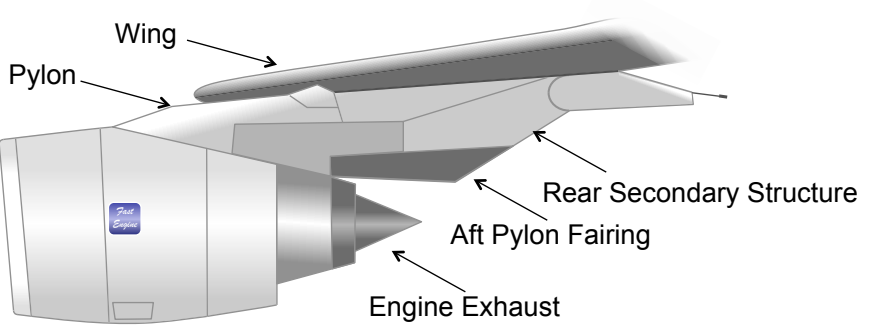

Figure 5. Localisation of the aft pylon fairing.

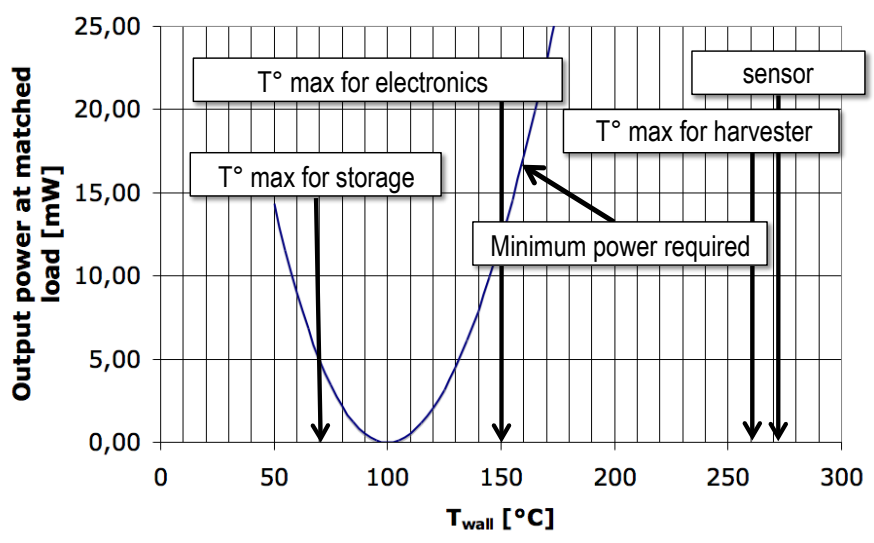

Figure 6. Example of coupled requirements for the different components of the node. The sensor is here supposed to be submitted to a temperature of $270^{\circ} \mathrm{C}$. The continuous curve represents the output power of a TEG.
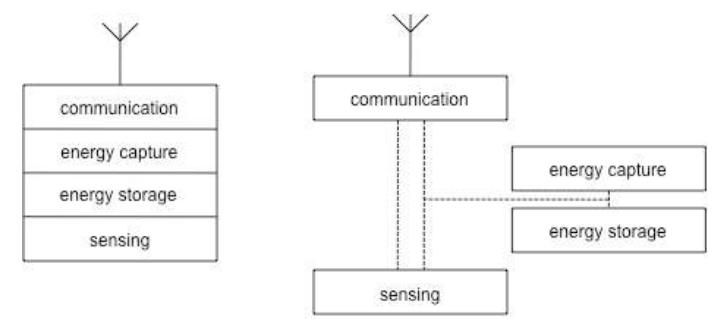

(2)

Figure 7. Wireless Sensor Network functional architecture. (1) all-in-one topology (2) split topology

\section{B. Photovoltaics}

For an aircraft manufacturer, flight-testing is essential to validate a design and obtain certifications from government agencies. Unfortunately, flight tests are expensive and complex at least for the wiring aspects as already illustrated in Fig. 2.

Among the typical measurements performed during flighttests, are pressure sensing at tens of positions around wings, tail, rudder... For this purpose, pressure gloves, themselves accommodating tens of sensors are affixed to the aircraft together with cables connecting them to data loggers (Fig. 8). This implies passing through multiple bulkheads and multiplying connectors. The replacement of this apparatus by WSN would reduce design load, installation and relocation time. Fortunately, for the above specific application, solar 
energy can be harvested because i) the sensors are located outside the aircraft ii) for security and simplicity reasons most flight tests are done at daylight.

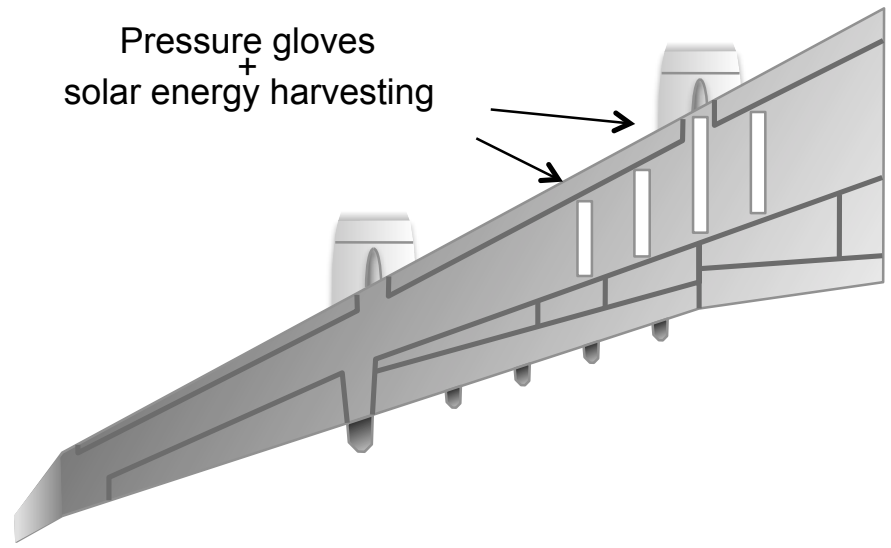

Figure 8. Schematic of pressure gloves affixed on top of a wing.

Solar energy is qualitatively one of the most abundant ambient energy and translates itself into electricity through mature and still progressing technology. However, specific requirements from the environment are two fold.

First the energy harvester (photovoltaic cells, maximum power point tracking circuit, energy management devices and buffer storage ultra capacitor) must be both very thin and globally flexible not to disturb the streamline flow. Considering areas, flexibility main impact is on photovoltaic cells, restricting the choice to less efficient cell technologies.

Then all devices must operate at extreme temperature (down to $-60^{\circ} \mathrm{C}$ ): fortunately low temperatures have a favorable impact on cells yield. On the other hand we identified manufacturers for buffer ultra capacitor, whose products perform well at such extreme temperatures.

Finally the amount of power delivered to the sensing and communicating devices was relatively high, given the high sampling frequency, number of sensor and wireless data rate.

Worth to mention is the fact that devising this system was easy as the environment (i.e. solar flux vs. altitude, time, season, location...) was initially well characterized, on the other hand the harvesting system (photovoltaic cells and associated electronics) was classical and mature, and because the objective was not to install a permanent system aiming at monitoring health of a critical structure during the airliner lifetime, but to deploy a temporary WSN.

\section{CONCLUSION}

In aeronautics, new needs call for the deployment of sensor networks. A lot of applications are related to SHM as illustrated above, but other areas such as flight-tests, aircraft security or logistic, are also demanding for new wireless and battery free communicating sensors. Whatever the application, environmental energy harvesting is then imperative to power new information processing systems.

It is the authors' opinion that in that context, flight-tests are likely to be the first field where wireless and energy autonomous sensors will be routinely deployed in the near future because of the gain induced by reduced design, complexity and installation time, by reason of the reduced number of connectors, on account of easy relocation, of the suppression of cables passing through multiple bulkheads, of the avoidance of rivet removal or hole drilling (an issue with composite materials). It is worth mentioning that in that context, the failure of one system has little consequence, the information lost during one test being accessible again during a future one. It is the authors' experience that for these temporary deployments, demand for battery free systems is indeed strong.

For permanent applications (such as SHM) autonomous energy generation will be considered only when a high number of sensors, in similar energetic situations, are envisioned. Conversely, if only a small number is needed, a power line is never that far in an aircraft to feed the devices and the complexity added by the extra wiring will be tolerable. In other words, the choice may be between a small number of wired sensors monitoring large areas, and a large number of wireless sensors with a more limited range. On the other hand, the issue of reliability and robustness of energy harvesters is here critical for applications such as SHM. Consequently, the application of WSN to SHM may be regarded as a long-term perspective.

Whatever the context, when solar energy is not possible and when efficient and reliable energy harvesters are required, TEGs will be one of the major competitors, benefiting from a large and for-years established commercial offer, combined with favorable intrinsic characteristics such as the absence of moving parts and maturity of technology.

\section{REFERENCES}

[1] N. Bailly, J-M. Dilhac, C. Escriba, C. Vanhecke, N. Mauran, M. Bafleur, "Energy Scavenging Based on Transient Thermal Gradients: Application to Structural Health Monitoring of Aircrafts," PowerMEMS 2008, Tokyo.

[2] D. Samson, M. Kluge, Th. Becker, U. Schmid, "Wireless Sensor Node Powered by Aircraft Specific Thermoelectric Energy Harvesting," Sensors and Actuators A / Physical, 2011.

[3] R. Montheard, C. Escriba, J.Y. Fourniols, M. Lastapis, J. Prunet, M. Bafleur, J.M. Dilhac, "Wireless and batteryless accelerometry for aircraft structural health monitoring," International Workshop on Structural Health Monitoring (IWSHM 2011), Stanford (USA), 2011.

[4] See for instance the International Workshop on Structural Health Monitoring Proceedings series which encompass aeronautics, maritime and civil engineering, and much more.

[5] European Commission, "Aeronautics and Air Transport: Beyond Vision $2020 ", 2010$.

[6] http://www.ultra-controls.com/productdetails.php?productID=21 